\title{
Comparison of the Explantation Rate of Poly Implant Prothèse, Allergan, and Pérouse Silicone Breast Implants within the First Four Years after Reconstructive Surgery before the Poly Implant Prothèse Alert by the French Regulatory Authority
}

\author{
Alexandre Leduey, ${ }^{1}$ Chafika Mazouni, ${ }^{1}$ Nicolas Leymarie, ${ }^{1}$ \\ Heba Alkhashnam, ${ }^{1}$ Benjamin Sarfati, ${ }^{1}$ Jean-Rémi Garbay, ${ }^{1}$ Amélie Gaudin, ${ }^{2}$ \\ Frédéric Kolb, ${ }^{1}$ and Françoise Rimareix ${ }^{1}$ \\ ${ }^{1}$ Department of Breast and Plastic Surgery, Gustave Roussy, 94805 Villejuif, France \\ ${ }^{2}$ Department of Pharmacology, Gustave Roussy, 94805 Villejuif, France \\ Correspondence should be addressed to Chafika Mazouni; chafika.mazouni@gustaveroussy.fr
}

Received 16 July 2015; Revised 16 September 2015; Accepted 27 September 2015

Academic Editor: Ian S. Fentiman

Copyright (C) 2015 Alexandre Leduey et al. This is an open access article distributed under the Creative Commons Attribution License, which permits unrestricted use, distribution, and reproduction in any medium, provided the original work is properly cited.

Background. In March 2010, ANSM (Agence Nationale de Sécurité du Medicament), the French Medical Regulatory Authority, withdrew Poly Implant Prothèse (PIP) breast implants from the market due to the use of non-medical-grade silicone gel. The aim of this study was to compare the removal rate (and reasons thereof) of breast implants produced by different manufacturers before the ANSM alert. Materials and Methods. From October 2006 to January 2010, 652 women received 944 implants after breast cancer surgery at the Gustave Roussy Comprehensive Cancer Center, Paris (France). The complications and removal rates of the different implant brands used (PIP, Allergan, and Pérouse) were evaluated and compared. Results. PIP implants represented 50.6\% of the used implants, Allergan 33.4\%, and Pérouse $16 \%$. The main reasons for implant removal were patient dissatisfaction due to aesthetic problems (43.2\%), infection (22.2\%), and capsular contracture (13.6\%). Two years after implantation, $82 \%$ of Pérouse implants, $79 \%$ of PIP, and $79 \%$ of Allergan were still in situ. There was no difference in removal rate among implant brands. Conclusion. Before the ANSM alert concerning the higher rupture rate of PIP breast implants, our implant removal rate did not predict PIP implant failure related to the use of nonapproved silicone gel.

\section{Introduction}

Immediate or secondary breast reconstruction can be performed using a breast implant alone or associated with a flap. Breast reconstruction with implants is almost the first choice for many women after breast cancer surgery because it allows rapid recovery, greater ability to change the breast volume, and avoidance of donor site morbidity $[1,2]$.

Breast implants have been developed and used for breast reconstruction for more than 40 years. Breast implants are manufactured as pliable silicone elastomer shells that are filled with silicone gel or saline solution. Silicone gel mimics the natural breast tissue more closely. Manufacturers have improved the implant safety and created many new models over the years. The last generation of implants has a multilayer shell with a barrier layer and is filled with thick silicone gel [3]. Many studies on the rate of complications, especially the rupture of silicone breast implants, during aesthetic or reconstructive surgery have demonstrated that silicone implants are safe and effective [4-7].

In early 2010, ANSM (Agence Nationale de Securité du Médicament), the French Medical Regulatory Authority, suspended the marketing, distribution, and use of silicone gel-filled breast implants produced by Poly Implant Prothèse (PIP) due to the use of a nonapproved filler material. Moreover, these breast implants did not have any elastomeric 
shell and the gel could more easily ooze through an intact implant shell and cause local irritation [8-11].

ANSM recommendation to the medical community was to monitor women with a PIP implant by ultrasound scan every six months to identify any implant rupture. At that time, systematic removal of PIP breast implants was not endorsed in the absence of conclusive scientific evidence concerning the gel toxicity [12]. However, on December 23, 2011, the French Ministry of Health recommended prophylactic surgery to remove PIP implants.

In the interval between the ANSM alert and the final recommendation about prophylactic surgery, the management of patients with PIP implants was left to the surgeon's discretion. Due to the growing media and patients' anxiety [13], the surgical team at the Gustave Roussy Comprehensive Cancer Center, Paris (France), decided to prophylactically remove all PIP breast implants.

A previously published study on systematic PIP implant removal after the ANSM alert reported high rates of breast implant leakage, but no rupture, with an average PIP breast implant lifespan of 21 months [14]. Another retrospective study to determine the rupture and complication rates of PIP breast implants found a high rate of implant rupture (7.7\%) associated with periprosthetic effusion in $44 \%$ of these patients [15]. However, no study has demonstrated that the rate of PIP breast implant complications is higher than that of other brands during the early years after implantation. Here, we evaluated and compared the rate of and reasons for removal of PIP, Allergan, and Pérouse breast implants (the three main breast implants brands used in our center) before the ANSM alert to determine whether any significant clinical events could have predicted the corporate fraud.

\section{Patients and Methods}

For this study, we retrospectively evaluated the data concerning all women who underwent breast reconstruction with breast implants at the Gustave Roussy Comprehensive Cancer Center (Paris, France) from 2006 to 2010. During this study period, PIP was the most frequently used breast implant.

Information on the implant brand, manufacturer, lot number, and lifespan was retrieved, after excluding all patients who had expander-based breast reconstruction and those who received Sebbin breast implants, which represented only $1 \%$ of the implants and were samples given by the manufacturer to be tested. We also identified the implantation date and all breast implant explantations carried out during the study period.

The breast implant lifespan by brand was determined before the ANSM alert and the prophylactic removal of PIP implants.

We also identified and recorded the causes leading to additional surgery and implant replacement. Causes of premature implant removal included, but were not limited to, rupture, capsular contracture, pain, distortion, rippling and wrinkling, infection, delayed healing with or without implant exposure, and patient dissatisfaction.

Quantitative data were compared using Pearson's chisquare and Fischer's exact tests. Factors predictive of implant
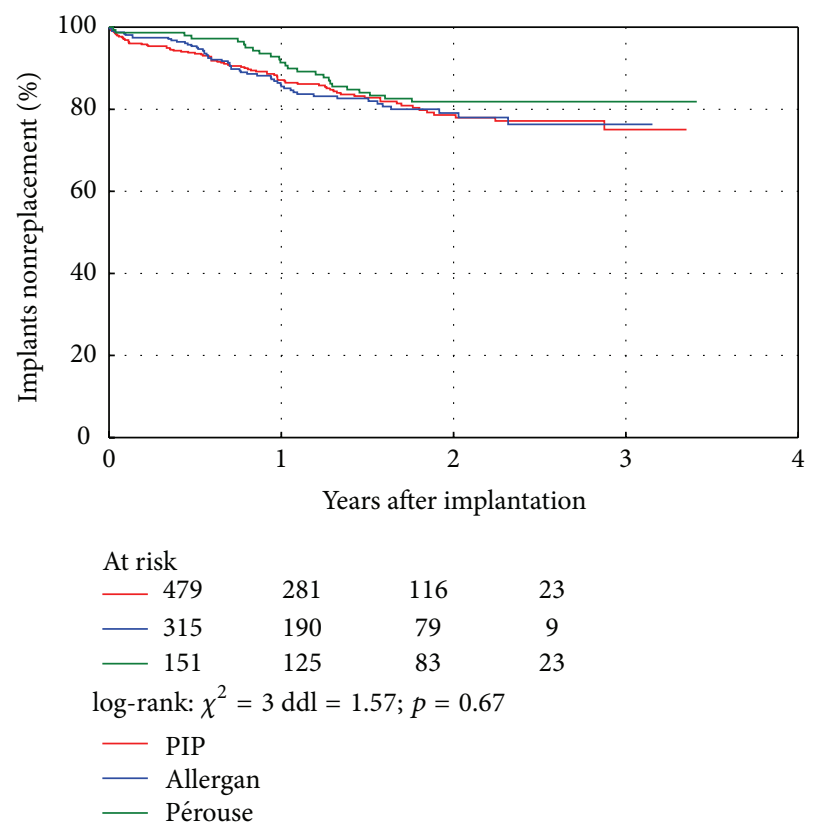

FIGURE 1: Breast implant longevity.

removal were analyzed using a logistic regression model and presented as odds ratios (OR) with their $95 \%$ confidence interval ( $95 \% \mathrm{CI})$. The lifespan of the different implant brands was compared using the Kaplan-Meier method. A $p$ value $<$ 0.5 was considered statistically significant.

\section{Results}

During the study period (between 2006 and 2010), 652 patients received a total of 944 breast implants (14\% of patients had bilateral breast reconstruction) following breast cancer surgery. The most used breast implants were PIP implants $(50.6 \% ; n=478)$, especially because of their asymmetric shape, followed by Allergan silicone gel implants $(33.4 \% ; n=315)$ and then Pérouse silicone gel implants (16\%; $n=151)$. These implant-based breast reconstructions were carried out by six surgeons.

Immediate breast reconstruction was the main indication for breast implant (53\%), followed by secondary breast reconstruction (29\%), contralateral breast augmentation (13\%), and implant exchange to improve breast shape (4\%). All implants were textured, $91 \%$ were asymmetric, and only $9 \%$ were round. All implants were placed in a subpectoral position.

Two years after implantation, $82 \%$ of Pérouse implants and 79\% of PIP and Allergan implants were still in situ (Figure 1). There was no difference between the brands in terms of implant lifespan $(p=0.67)$.

Overall, 158 of the 944 breast implants (16.7\%) were removed: 81 were PIP implants (17\% of all PIP implants), 52 were Allergan implants (16.5\%), and 25 were Pérouse implants (16.5\%). The implant removal rate was not statistically different among the three brands $(p=0.43)$. The average time to explantation was 299 days (4-1050 days). 
TABLE 1: Reasons for breast implant removal.

\begin{tabular}{|c|c|c|c|c|c|}
\hline Complications & PIP (81) & Allergan/Pérouse (77) & OR & $95 \% \mathrm{CI}$ & $p$ \\
\hline Infection & $18(22.2)$ & $8(10.4)$ & 2.45 & {$[0.9 ; 6.9]$} & 0.054 \\
\hline Exposure & $5(6.2)$ & $5(6.5)$ & 1.05 & {$[0.2 ; 4.7]$} & 1 \\
\hline Rupture & $4(4.9)$ & $0(0)$ & - & {$[0.63 ;-]$} & 0.12 \\
\hline Capsular contracture & $11(13.6)$ & $17(22.1)$ & 0.59 & {$[0.2 ; 1.5]$} & 0.3 \\
\hline Aesthetic reoperation & $35(43.2)$ & $37(48.1)$ & 1.05 & {$[0.5 ; 2.1]$} & 1 \\
\hline Migration & $2(2.5)$ & $6(7.8)$ & 0.3 & {$[0.03 ; 1.8]$} & 0.16 \\
\hline Cancer recurrence & $2(2.5)$ & $1(1.3)$ & 1.9 & {$[0.1 ; 114.9]$} & 1 \\
\hline Others & $4(4.9)$ & $3(3.9)$ & 1.2 & {$[0.2 ; 9.03]$} & 1 \\
\hline Number (\%) & $81(100)$ & $77(100)$ & 0.97 & {$[0.7 ; 1.4]$} & 0.93 \\
\hline
\end{tabular}

OR: odds ratio; CI: confidence interval.

During the study period (before the ANSM alert), PIP implants were removed for the following reasons (Table 1): unsatisfactory aesthetic results that required implant replacement $(n=35$ patients), infection that required immediately further surgery ( $n=18$ patients), implant exposure ( $n=5$ patients), suspected rupture that was confirmed intraoperatively ( $n=4$ patients), capsular contraction $(n=$ 11 patients), migration or rotation of the implant $(n=2$ patients), or recurrent cancer $(n=2)$. Comparison of the causes that led to implant replacement did not highlight any significant difference between PIP and Allergan/Pérouse implants (Table 1), although the infection rate tended to be higher $(p=0.054)$ in the PIP group.

\section{Discussion}

Our study compared the rate and causes of PIP implant replacement with that of other implant brands used in our center (Allergan and Pérouse) during the first years after implantation. Our objective was to determine whether some undesirable events could have alerted surgeons before the ANSM alert about the higher rupture rate among PIP breast implants on March 29, 2010 [8].

Since the introduction of the first breast implants in the 1960s, their design and manufacturing have changed radically with the arrival of cohesive gels, thick prosthetic membranes, textured surfaces, and improved quality control. The current breast implants offer better safety with less implant rotation and less capsular contracture $[6,16]$. This qualitative improvement was the reason for their return to the French market in 2001 after being banned for several years. Cohesive gel implants are considered to be the undisputed gold standard for breast reconstruction. Breast implant factories are now regularly inspected and adverse events must be declared to the ANSM.

The abnormal increase in the rupture rate of PIP implants led to more frequent inspections of the PIP implant factories. This revealed serious shortcomings in the manufacturing of these products. The silicone gel used was not of medical grade with a low polymerization rate and many short chain residues that leak more easily through the implant shell. This side effect was even more a cause for concern because it reduces the efficacy of the implant shell by increasing its porosity $[9,11]$.
Between 2006 and 2010 most of the implants fitted in our surgery department were PIP products (50.6\% of patients with breast reconstruction had a PIP implant fitted). Before a medical device is available in our institution, it has to be referenced by the French Federation of Cancer Centers (UNICANCER), based on the evaluation of its safety and technical advantages compared to the already used material. In 2006, PIP implants were introduced into our department because they offered a larger base width and an anatomical shape that gave a more natural breast for reconstruction, compared to other brands [16]. Therefore, we began to use them frequently due to their advantages in terms of shape and final aesthetic results.

The incidence of implant failure is generally low [17, 18]. In our population, $16.7 \%$ of patients needed an implant replacement before four years. We did not find significant differences between the rates of PIP removal and that of the other brands. Interestingly, no report about an abnormal removal rate of PIP implants was published before the ANSM alert. Moreover, the major causes of breast implant removal were not related to the gel mechanical and viscoelastic properties, but to aesthetic revision, capsular contracture, and infection, like for the other brands. The similar fouryear reoperation rate due to aesthetic problems for the three brands indicates that the mechanical and viscoelastic properties of the gel used for the PIP implants did not influence the aesthetic result. However, previous studies reported the anomalous early tendency of PIP implants to disintegrate shortly after implantation and the inflammatory response caused by the leakage of silicone gel [19-22]. Our study highlighted a trend towards a higher infection rate with PIP implants $(p=0.054)$ for which we have no explanation. Bacterial culture was not carried out in all cases of infection and antibiotics were administered in the presence of clinical signs of inflammatory reactions. For all implants, the body naturally forms more or less tight capsules that could give rise to capsular contracture, leading to their removal. Most capsular contractures are observed during the early years after implant placement. Our results suggest that the rate of capsular contractures is not affected by the biomedical quality of the silicone gel, but more by the choice of the pocket and the implant surface [23].

Breast implant removal due to implant rupture within the first four years after surgery concerned $4.9 \%$ of the patients 
with a PIP implant compared to $0 \%$ for the other brands. In 2006 , Hedén et al. reported $6 \%(n=199)$ of ruptured implants during a mean follow-up period of 10.9 years and only $0.3 \%$ of ruptures during the first five years [24]. The implant rupture rate ranges from 0.3 to $77 \%$ in the literature [24-30] due to the implant heterogeneity and manufacturing changes during recent years. The risk is cumulative and is estimated to be approximately $6 \%$ per year during the first five years following the primary implant surgery [29]. Our failure rate was low: $0.8 \%$ at four years for all implants. The rupture rate of PIP silicone implants (4.9\%) was not significantly higher compared to that of other brands (only four PIP implant ruptures). This rate is low and was based on removal due to clinical suspicion of implant rupture before the systematic monitoring/explantation of PIP breast implant after the ANSM alert. Therefore, the rupture rate in our series of patients with breast cancer was probably underestimated, compared to the rupture rate (16.1\%; 4130/25644 implants) after systematic explantation of PIP implants reported by ANSM [31]. Even higher PIP rupture rates $(30.9 \%$ in 2000 and $35.1 \%$ in 2001) were observed by Maijers and Niessen [32]. Moreover, the ANSM reported an overall rate of implant failures (rupture, gel bleeding, and capsule contraction) or other adverse events of $21 \%$ after preventive explantation.

The PIP implant biodurability was related to the implantation year, with a median time to rupture of 10.5 years in 2000 and 5.8 years in 2005, showing that PIP implants were becoming less durable [33]. Recent studies suggest that the use of medically unapproved silicone gel in the PIP implants did not contribute to their rupture [32], which was mainly caused by the shell weakness or shell failure due to different variables, including variable shell thickness, the nature of shell texturing, sharp corners, and identification marks [11].

We did not evaluate the rupture rate after the ANSM alert in 2010. Although our study reveals that the rupture rate of PIP implants was not significantly different from that of other brands, we must not forget that the silicone gel used was not approved by the regulatory medical authority. We therefore agree with the recommendation that PIP implants should be removed prophylactically. Besides the physical danger of these implants, there is also an important psychological factor, especially in patients who had been treated for breast cancer and who should be spared additional worries.

\section{Conclusion}

Before the discovery that medically unapproved silicone gel was used in PIP breast implants, the reasons for implant removal would not have allowed us to unmask the manufacturer's fraud. Indeed, implant rupture was not significantly more frequent with PIP than with Allergan or Pérouse breast implants.

\section{Conflict of Interests}

The authors declare that there is no conflict of interests regarding the publication of this paper.

\section{References}

[1] J. F. Delgado, R. F. García-Guilarte, M. R. Palazuelo, J. I. S. Mendez, and C. C. Pérez, "Immediate breast reconstruction with direct, anatomic, gel-cohesive, extra-projection prosthesis: 400 cases," Plastic and Reconstructive Surgery, vol. 125, no. 6, pp. 1599-1605, 2010.

[2] J. Roostaeian, L. Pavone, A. Da Lio, J. Lipa, J. Festekjian, and C. Crisera, "Immediate placement of implants in breast reconstruction: patient selection and outcomes," Plastic and Reconstructive Surgery, vol. 127, no. 4, pp. 1407-1416, 2011.

[3] M. Johnson, "Breast implants: history, safety, and imaging," Radiologic Technology, vol. 84, pp. 439M-520M, 2013.

[4] L. A. Brinton, L. M. Buckley, O. Dvorkina et al., "Risk of connective tissue disorders among breast implant patients," American Journal of Epidemiology, vol. 160, no. 7, pp. 619-627, 2004.

[5] H. Shaaban and R. Alvi, "Locoregional silicone spread after high cohesive gel silicone implant rupture," Journal of Plastic, Reconstructive \& Aesthetic Surgery, vol. 60, no. 10, pp. 1166-1167, 2007.

[6] J. K. McLaughlin, L. Lipworth, D. K. Murphy, and P. S. Walker, "The safety of silicone gel-filled breast implants: a review of the epidemiologic evidence," Annals of Plastic Surgery, vol. 59, no. 5, pp. 569-580, 2007.

[7] K. C. Tark, H. S. Jeong, T. S. Roh, and J. W. Choi, "Analysis of 30 breast implant rupture cases," Aesthetic Plastic Surgery, vol. 29, no. 6, pp. 460-471, 2005.

[8] MHRA medical device alert MDA/2010/025, 2010.

[9] G. Beretta and M. Malacco, "Chemical and physicochemical properties of the high cohesive silicone gel from Poly Implant Prothèse (PIP) breast prostheses after explantation: a preliminary, comparative analytical investigation," Journal of Pharmaceutical and Biomedical Analysis, vol. 78-79, pp. 75-82, 2013.

[10] A. Daniels, "Silicone breast implant materials," Swiss Medical Weekly, vol. 142, Article ID w13614, 2012.

[11] E. Swarts, A. M. Kop, A. Nilasaroya, C. V. Keogh, and T. Cooper, "Rupture of poly implant prothèse silicone breast implants: an implant retrieval study," Plastic and Reconstructive Surgery, vol. 131, no. 4, pp. 480e-489e, 2013.

[12] AFSSAPS Medical Devices Evaluation Direction, Silicone Based Filling Gel Breast Implants from Poly Implant Prothèse Company: Update of Test Results, Agence Française de Sécurité Sanitaire des Produits de Santé, 2011.

[13] M. G. Berry and J. J. Stanek, "PIP implant biodurability: a postpublicity update," Journal of Plastic, Reconstructive and Aesthetic Surgery, vol. 66, no. 9, pp. 1174-1181, 2013.

[14] C. Crouzet, D. Gangloff, B. Chaput, J.-L. Grolleau, and I. Garrido, "Outcome at 18 months of the recall Poly Implant Prosthesis implants. Experience of a cancer center," Annales de Chirurgie Plastique Esthétique, vol. 57, no. 1, pp. 9-15, 2012.

[15] S. Oulharj, J. Pauchot, and Y. Tropet, "PIP breast implant removal: a study of 828 cases," Journal of Plastic, Reconstructive and Aesthetic Surgery, vol. 67, no. 3, pp. 302-307, 2014.

[16] T. F. Henriksen, L. R. Hölmich, J. P. Fryzek et al., "Incidence and severity of short-term complications after breast augmentation: results from a nationwide breast implant registry," Annals of Plastic Surgery, vol. 51, no. 6, pp. 531-539, 2003.

[17] C. M. Robbins, J. N. Long, R. J. Fix, J. I. de La Torre, and L. O. Vasconez, "Mastectomy with breast reconstruction in previously augmented patients: indications for implant removal," Annals of Plastic Surgery, vol. 61, no. 5, pp. 500-505, 2008. 
[18] G. P. Maxwell, B. W. Van Natta, D. K. Murphy, A. Slicton, and B. P. Bengtson, "Natrelle style 410 form-stable silicone breast implants: core study results at 6 years," Aesthetic Surgery Journal/The American Society for Aesthetic Plastic surgery, vol. 32, no. 6, pp. 709-717, 2012.

[19] R. B. Berry, "Rupture of PIP breast implants," Journal of Plastic, Reconstructive and Aesthetic Surgery, vol. 60, no. 8, pp. 967-968, 2007.

[20] U. D. Khan, "Left unilateral breast autoinflation and intraprosthetic collection of sterile pus: an unusual operative finding of silicone gel bleed with silicone lymphadenitis," Aesthetic Plastic Surgery, vol. 32, no. 4, pp. 684-687, 2008.

[21] U. D. Khan, "Breast autoinflation with sterile pus as a marker of implant rupture: single-stage treatment and outcome for five consecutive cases," Aesthetic Plastic Surgery, vol. 33, no. 1, pp. 58-65, 2009.

[22] A. Lahiri and R. Waters, "Locoregional silicone spread after high cohesive gel silicone implant rupture," Journal of Plastic, Reconstructive and Aesthetic Surgery, vol. 59, no. 8, pp. 885-886, 2006.

[23] T. F. Henriksen, J. P. Fryzek, L. R. Hölmich et al., "Surgical intervention and capsular contracture after breast augmentation: a prospective study of risk factors," Annals of Plastic Surgery, vol. 54, no. 4, pp. 343-351, 2005.

[24] P. Hedén, M. B. Nava, J. P. van Tetering et al., "Prevalence of rupture in inamed silicone breast implants," Plastic \& Reconstructive Surgery, vol. 118, no. 2, pp. 303-312, 2006.

[25] O. G. Robinson Jr., E. L. Bradley, and D. S. Wilson, "Analysis of explanted silicone implants: a report of 300 patients," Annals of Plastic Surgery, vol. 34, no. 1, pp. 1-7, 1995.

[26] S. L. Brown, M. S. Middleton, W. A. Berg, M. S. Soo, and G. Pennello, "Prevalence of rupture of silicone gel breast implants revealed on MR imaging in a population of women in Birmingham, Alabama," American Journal of Roentgenology, vol. 175, no. 4, pp. 1057-1064, 2000.

[27] S. L. Brown, G. Pennello, W. A. Berg, M. S. Soo, and M. S. Middleton, "Silicone gel breast implant rupture, extracapsular silicone, and health status in a population of women," Journal of Rheumatology, vol. 28, no. 5, pp. 996-1003, 2001.

[28] J. S. Marotta, C. W. Widenhouse, M. B. Habal, and E. P. Goldberg, "Silicone gel breast implant failure and frequency of additional surgeries: analysis of 35 studies reporting examination of more than 8000 explants," Journal of Biomedical Materials Research, vol. 48, no. 3, pp. 354-364, 1999.

[29] J. S. Marotta, E. P. Goldberg, M. B. Habal et al., "Silicone gel breast implant failure: evaluation of properties of shells and gels for explanted prostheses and meta-analysis of literature rupture data," Annals of Plastic Surgery, vol. 49, no. 3, pp. 227-247, 2002.

[30] L. R. Hölmich, K. Kjøller, I. Vejborg et al., "Prevalence of silicone breast implant rupture among Danish women," Plastic and Reconstructive Surgery, vol. 108, no. 4, pp. 848-863, 2001.

[31] PIP Breast Implants, Situation update. April 2013, http:// ansm.sante.fr/var/ansm_site/storage/original/application/ ea94f5f3532f4f831d6a923ef553a77e.pdf.

[32] M. C. Maijers and F. B. Niessen, "Prevalence of rupture in poly implant prothèse silicone breast implants, recalled from the European market in 2010," Plastic and Reconstructive Surgery, vol. 129, no. 6, pp. 1372-1378, 2012.

[33] M. G. Berry and J. J. Stanek, "The poly implant prothèse debacle," Plastic and Reconstructive Surgery, vol. 131, no. 1, p. $110 \mathrm{e}-2 \mathrm{e}, 2013$. 


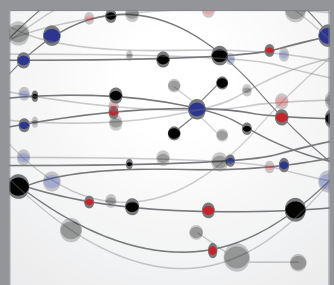

The Scientific World Journal
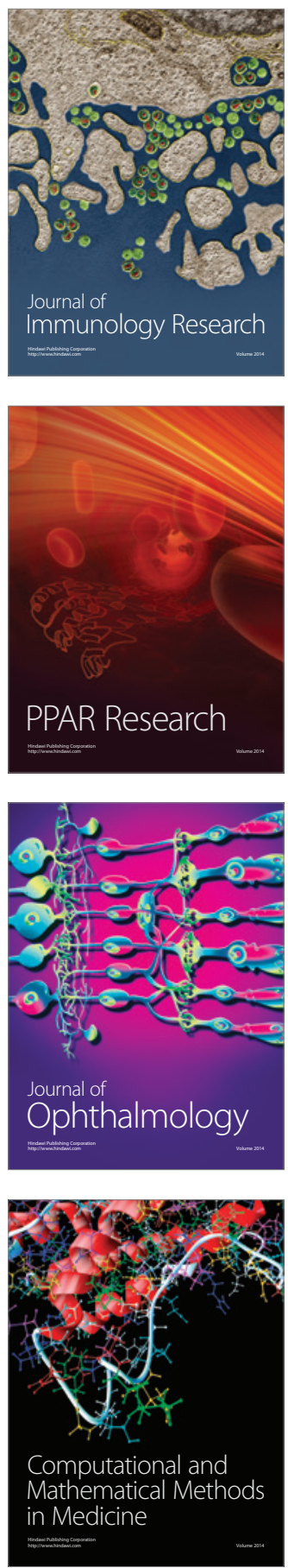

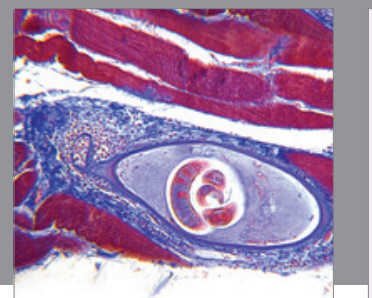

Gastroenterology

Research and Practice
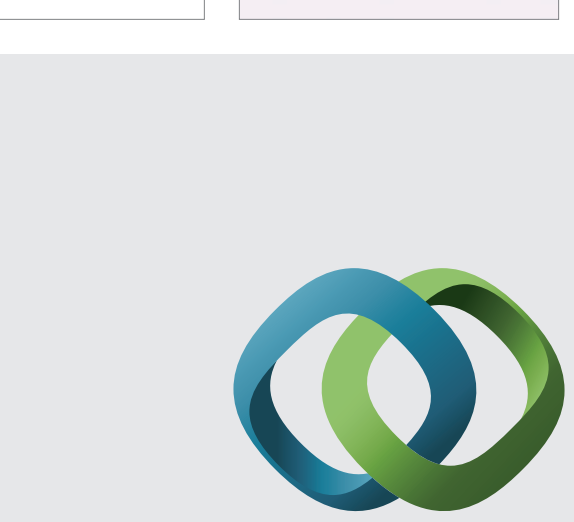

\section{Hindawi}

Submit your manuscripts at

http://www.hindawi.com
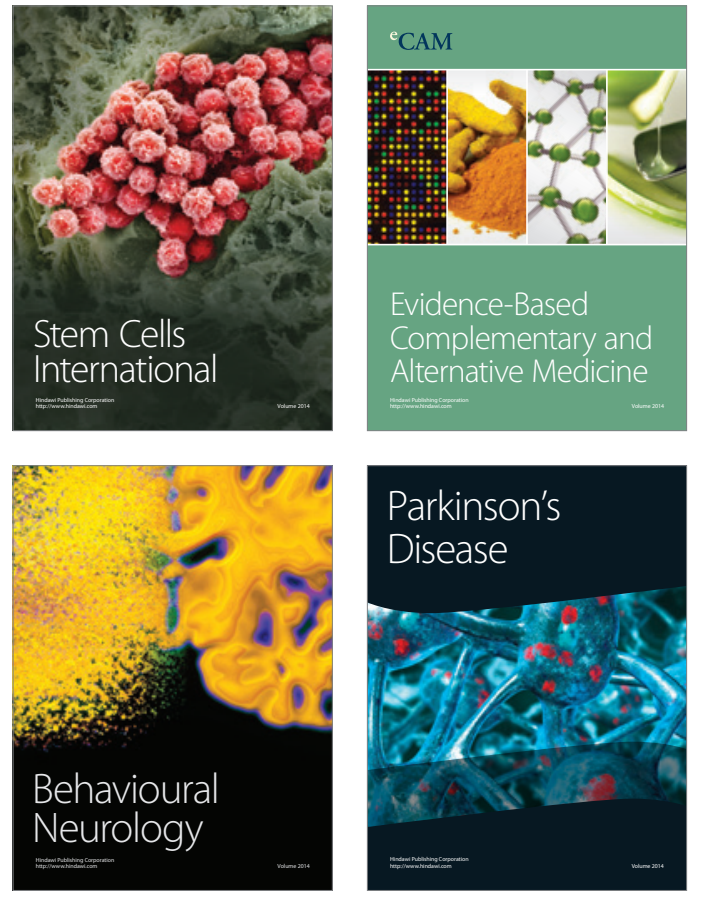
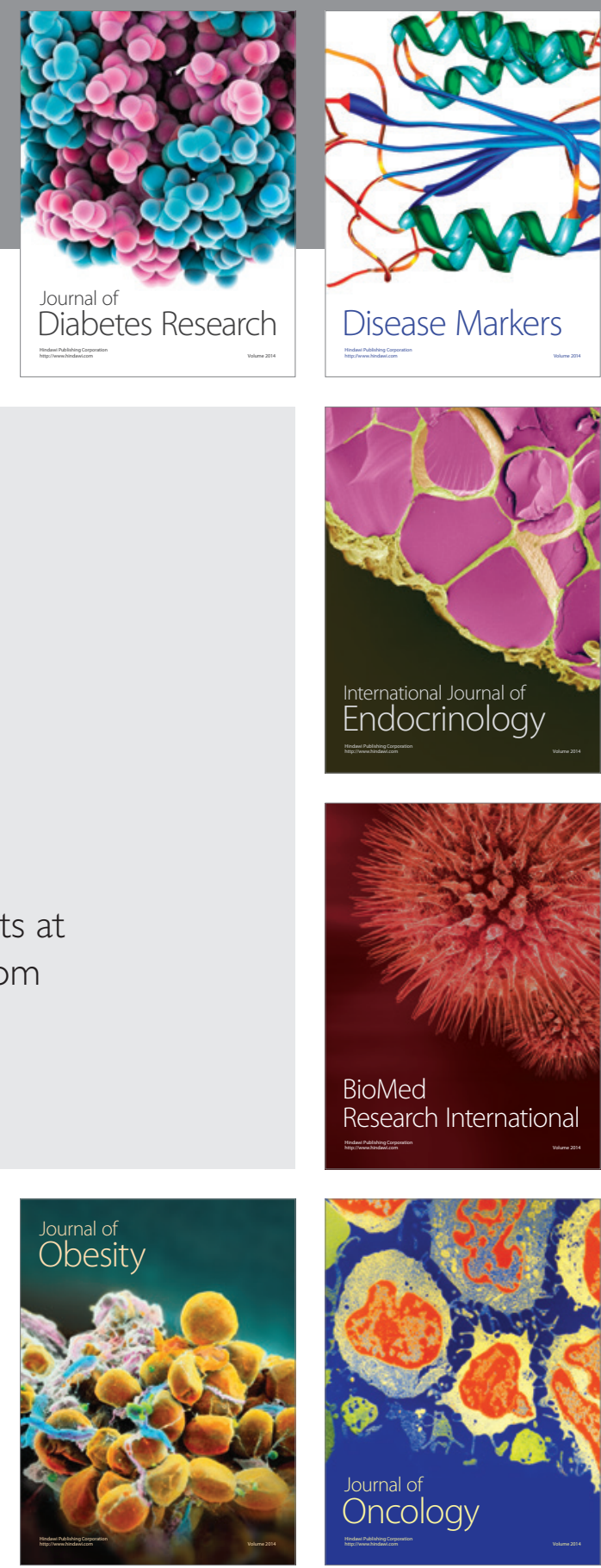

Disease Markers
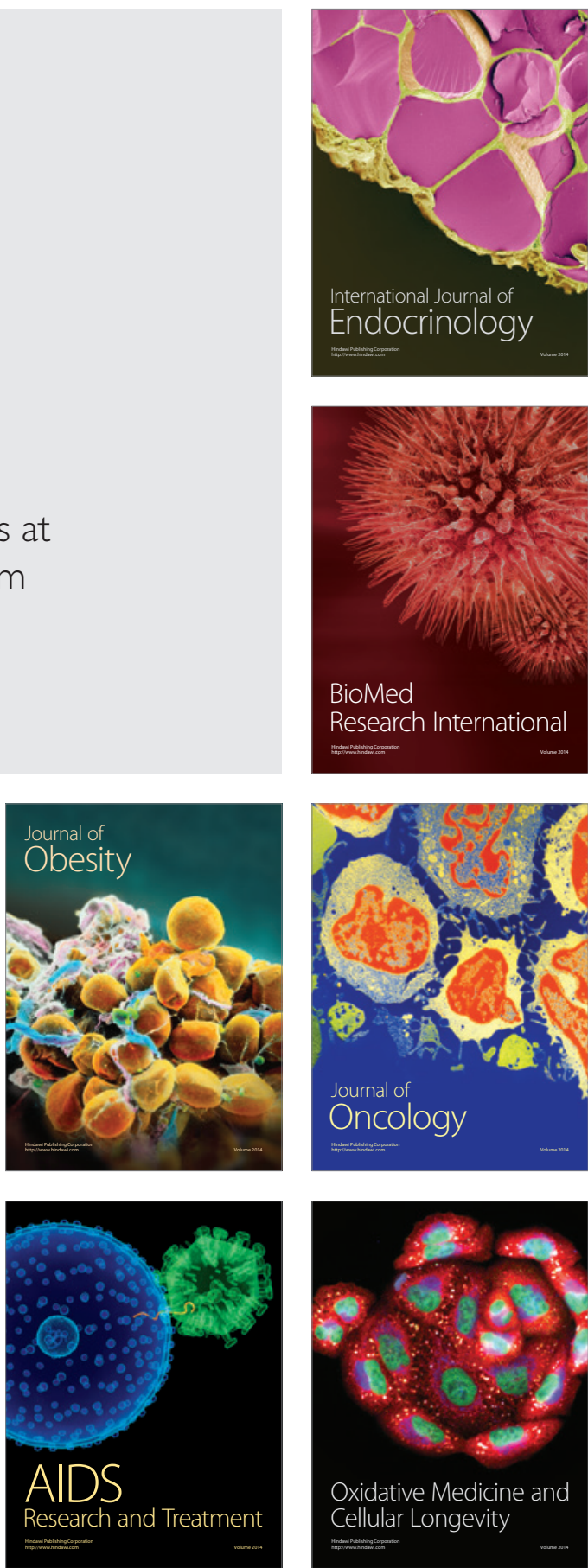